\title{
Associations between the Presence of Granulomata and Disease Phenotype and Outcomes in Children Diagnosed with Crohn's Disease
}

\author{
Laura Appleton ${ }^{1}{ }^{\circ}$, Euan Watt ${ }^{1,2}$, Fiona Jagger ${ }^{2}$, Richard Hansen ${ }^{3}{ }^{\oplus}$, Richard B. Gearry ${ }^{4}$ and \\ Andrew S. Day ${ }^{1, *,+}$ (D) \\ 1 Department of Paediatrics, University of Otago Christchurch, Christchurch 8011, New Zealand; \\ laura.appleton@otago.ac.nz (L.A.); erw269@gmail.com (E.W.) \\ 2 School of Medicine \& Dentistry, University of Aberdeen, Aberdeen AB24 3FX, Scotland, UK; fjagger@nhs.net \\ 3 Department of Paediatric Gastroenterology, Royal Hospital for Children, Glasgow G51 4TF, Scotland, UK; \\ richard.hansen@nhs.net \\ 4 Department of Medicine, University of Otago Christchurch, Christchurch 8011, New Zealand; \\ richard.gearry@cdhb.health.nz \\ * Correspondence: andrew.day@otago.ac.nz; Tel.: +64-3-372-6718 \\ + Cure Kids Chair of Paediatric Research, Department of Paediatrics, University of Otago, \\ Christchurch 4 Riccarton Avenue, Christchurch 8140, New Zealand.
}

Received: 15 May 2020; Accepted: 10 June 2020; Published: 13 June 2020 updates

\begin{abstract}
Background: The finding of a mucosal granuloma on histological analysis of endoscopically obtained biopsies in children with Crohn's disease has been suggested to provide prognostic information. The aim of this study was to retrospectively assess the rate of granuloma detection and the impact of this upon specific disease characteristics and outcomes in children diagnosed with Crohn's disease. After identification of a group of children previously diagnosed with Crohn's disease, chart reviews were undertaken to characterise the children as granuloma positive or negative. Disease characteristics at diagnosis (such as disease location and nutritional status) and following diagnosis (such as requirement for immunosuppressive medications and surgical intervention) were noted for each patient. Results: Ninety-four children from two distinct geographical areas were identified. Forty-nine (52.1\%) of the children had mucosal granulomata. Children with colonic disease were likely to have granulomata detected $(\mathrm{RR}=3.04 ; p<0.001)$. Granulomata were associated with lower weight $z$-scores at diagnosis $(p<0.05)$, but not other disease features (e.g., perianal disease or extra-intestinal manifestations). The presence of a granuloma at diagnosis was also associated with increased rates of the subsequent requirement for an immunosuppressive medication $(\mathrm{RR}=1.26$; $p=0.002$ ). The presence of granulomata on histological assessment of mucosal biopsies at diagnosis of children with Crohn's disease appears to be associated with specific disease features and outcomes. These findings should be clarified prospectively in a larger cohort of children with Crohn's disease.
\end{abstract}

Keywords: Crohn's disease; granuloma; children; nutrition; disease outcomes

\section{Introduction}

Crohn's disease (CD), one of the main subtypes of inflammatory bowel disease (IBD), is thought to be caused by a complex interaction of genetic susceptibility, environmental factors and immunological defects [1,2]. CD may involve any section of the gastrointestinal tract and commonly presents with gastrointestinal symptoms, such as abdominal pain, diarrhoea and weight loss. Diagnosis of CD is based on endoscopic, histologic and radiological findings, with characteristic features being transmural inflammation, skip lesions and mucosal non-caseating granuloma $[1,2]$. 
While the peak age at diagnosis of CD is in early adult years, up to $25 \%$ of all new diagnoses occur within the paediatric population [3,4]. Paediatric-onset CD is typically more extensive with more involvement of the proximal gut than adult-onset CD [5,6]. In recognition of these differences, IBD in children and adolescents is classified using an independent system known as the Paris classification [7]. Treatment regimens typically also differ from those utilised in adults with CD: for example, international guidelines promote the use of exclusive enteral nutrition as the first-line therapy to induce remission after diagnosis of $\mathrm{CD}$ in children [8].

While the phenotype of $\mathrm{CD}$ in children and adolescents can be characterised by more extensive disease involvement, the patterns of disease phenotype and disease behaviour are not consistent throughout the paediatric population. Furthermore, there are no reliable methods to predict the likely disease course in an individual patient following diagnosis of CD. The ability to identify those patients who will have rapid disease progression or early disease complications could enable the use of more focused or aggressive therapies that might then modify the course of the disease. The presence or absence of granulomata in mucosal biopsies has been hypothesised to provide some prognostic information [9-11]. Although the histological presence of granulomata is considered virtually diagnostic of $C D$, they are only present in up to two thirds of children with $C D[10,12-14]$. This retrospective study aimed to assess the relevance of granulomata on disease phenotype and outcomes in two geographically distinct groups of children diagnosed with CD.

\section{Materials and Methods}

\subsection{Study Population}

The cohort comprised children (aged less than 18 years) diagnosed with CD at Christchurch Hospital (Christchurch, New Zealand) and NHS Grampian (Scotland, United Kingdom). The diagnosis of $\mathrm{CD}$ was based upon standard criteria and followed upper gastrointestinal endoscopy, colonoscopy and small bowel imaging [7]. Age at diagnosis was defined as the date of diagnostic endoscopic assessment.

Inclusion criteria for this analysis was a full set of demographic and clinical data that included age at diagnosis, gender, disease location and severity at diagnosis, and histological data. The distribution of CD involvement was classified according to the Paris classification system. Disease behaviour (inflammatory or complicated disease) was recorded as the worst ever over the period of observation. The pattern of symptoms at presentation and recording of family history of IBD were noted, where available. Nutritional status (weight and height) at diagnosis were converted to z-scores for age using the National Centre for Health Statistics 2000 growth data (Centers for Disease Control and Prevention, Atlanta, GA, USA).

\subsection{Data Management}

Upon identification of eligible patients on existing databases in both centres, their paper and electronic medical records were reviewed. Databases were created on Microsoft Excel using an encrypted hard drive. Study identification numbers were utilised throughout to maintain anonymity. Local ethics approval at either centre was not required for this low-risk study as it did not involve direct patient contact and comprised a retrospective audit of clinical outcomes.

For each patient, granuloma status was recorded as either positive or negative. Positive status was defined as a confirmative pathology report of mucosal biopsies obtained at diagnostic endoscopy containing one or more granulomata.

\subsection{Disease Outcome Parameters}

Major clinical outcomes were identified and analysed for each patient: these included clinical relapse and hospital admissions (calculated as events per year since diagnosis), the need for second 
line treatment (biologics and immunosuppressive therapy), the need for surgical intervention and nutritional status.

A clinical relapse was defined as any time the patient had an exacerbation of symptoms requiring assessment and/or intervention based upon available clinic letters and discharge notes. A hospital admission was defined as any overnight hospital stay for management of an exacerbation or complication of $\mathrm{CD}$.

The use of an immunomodulator or biological therapy was noted for each patient and outcomes expressed as "ever used" or "not". Surgical intervention was defined as any documented procedure to resect an area of the gut for the management of the patient's CD. Any surgical procedure for management of manifestations such as anal fissures or skin tags was excluded.

\subsection{Data Analysis}

SPSS was employed for analysis of data (IBM SPSS Statistics for Windows, version 25 (IBM Corp, Armonk, NY, USA). Categorical data were analysed using Chi-squared and Fisher's exact test (when expected value in any cell was less than 5). Contingency tables were used to calculate relative risks and Kruskal-Wallis test was utilised for between group comparisons of numerical data. Significance was accepted as $p<0.05$.

\section{Results}

\subsection{Patient Population}

Ninety-four patients fitting the inclusion criteria were included (62 from New Zealand and 32 from Scotland). The median age (range) at diagnosis was 12.1 (2.3-17.8) years and the mean duration of follow up was $8.03(1-34)$ years (Table 1).

Table 1. Demographic and phenotype characterization for population of 94 children with Crohn's disease, according to the presence or absence of granulomata. NS = not significant.

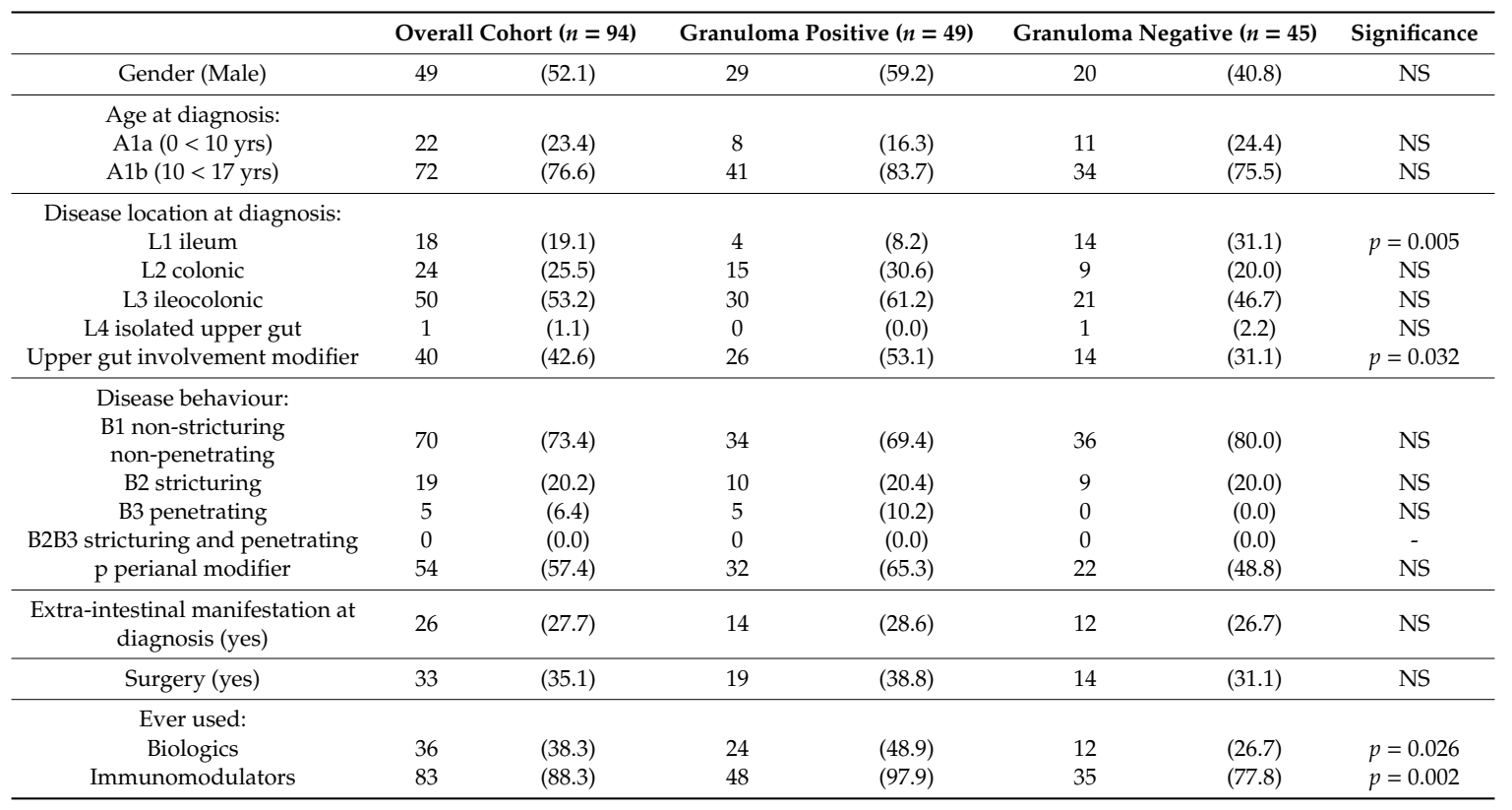

Granulomata were present at diagnosis in 49 (52.1\%) out of 94 patients. Age, gender, disease classification and granuloma distribution did not differ significantly between the two geographic groups. 


\subsection{Disease Phenotype and Outcomes According to the Presence or Absence of Granuloma}

When grouped by disease location alone, those with colonic involvement (L2 and L3) appeared to have a higher median relapse rate than non-colonic (L1, L4 only) (1 per year vs. 0.51 per year, respectively; $p=0.057$ ) (Figure 1 ). Children with colonic involvement were also younger at diagnosis than those without colonic involvement (mean age $=11.6$ years vs. 13.9 years; $p=0.006$ ).

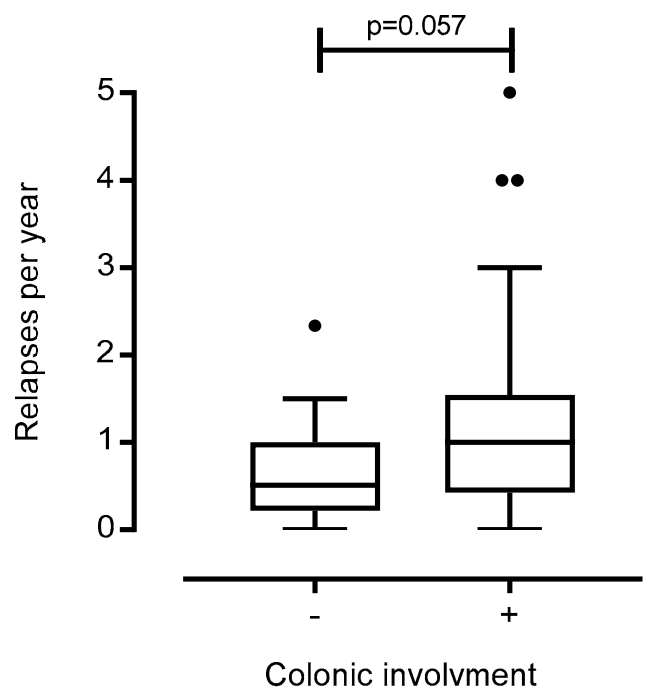

Figure 1. Distribution of relapses per year according to disease location (colonic vs. non-colonic) in children diagnosed with Crohn's disease (Turkey boxplot (1.5IQR) for outliers).

Granuloma positive and negative groups did not differ significantly in age, gender or for the presence of extra-intestinal manifestations (EIM), disease behaviour or the presence of perianal disease (Table 1). Patients were three times more likely to have granulomata if the colon was involved (L2 and L3) compared with patients with ileal (L1) or isolated upper gut disease (L4 only) (RR = 3.04; 95\% confidence interval (CI) 1.24-7.44; $p<0.001$ ).

There were no differences in the relapse rate, hospital admission rate and surgical risk between the two groups ( $p>0.05$; data not shown). However, patients with a granuloma were more likely to need second line treatments than those without a granuloma $(\mathrm{RR}=1.26 ; 95 \% \mathrm{CI} 1.07-1.48 ; p=0.002)$.

\subsection{Nutrition}

Anthropometric measurements were available for 79 patients (43 with granuloma). The median height z-score at diagnosis was $-0.568(-3.287$ to +2.702$): 69.6 \%$ of patients presented with below average height for their age at diagnosis. However, there was no significant difference between the median scores for the groups who did or did not have granulomata $(p>0.05)$ (Figure 2A). In contrast, $75.9 \%$ of patients were below average weight for their age at diagnosis: median weight $\mathrm{z}$-score was $-0.759(-3.785$ to +3.401$)$. The children with granulomata were more likely to be underweight than those without granulomata at diagnosis (z-scores $-0.9135(-3.785$ to +1.713$)$ vs. $-0.234(-2.82$ to 3.401 ; $p<0.05)$ ) (Figure 2B). 

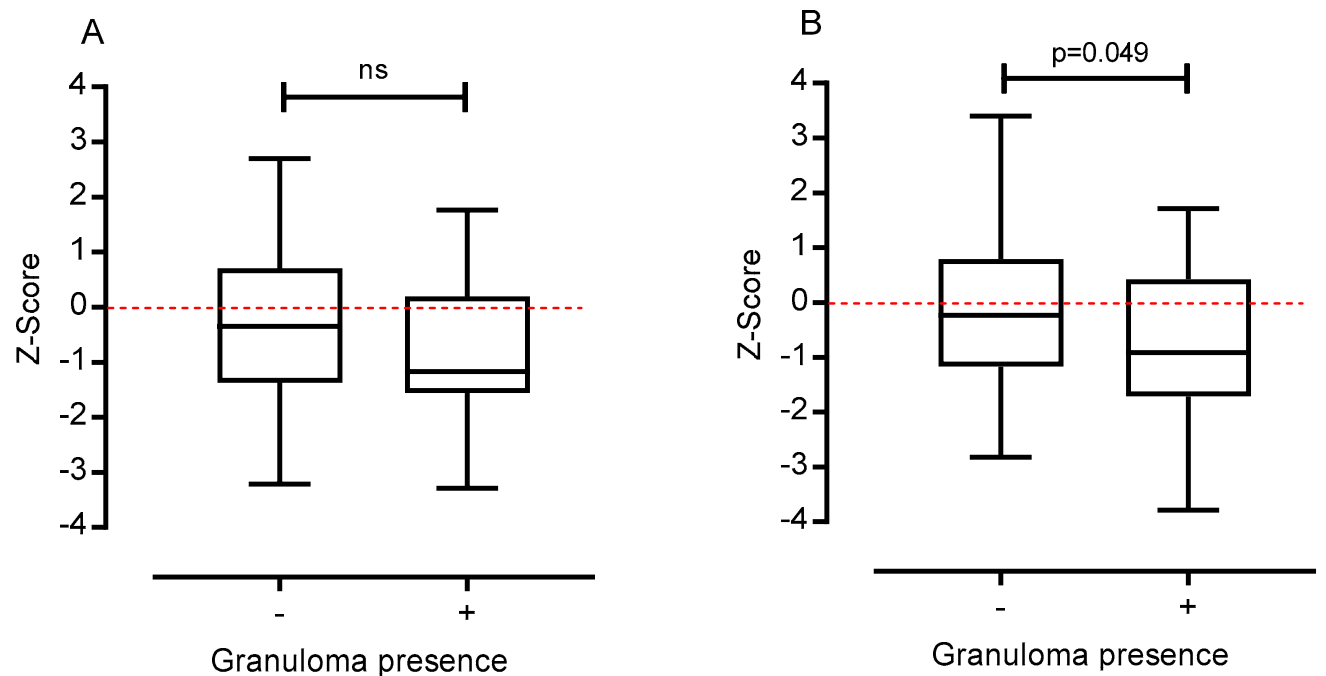

Figure 2. Anthropometric measurements at diagnosis of 79 children with Crohn's disease; z-score distribution of height (A) and weight (B) measurements of patients who were granuloma negative or positive $\mathrm{ns}=$ not significant

\section{Discussion}

This retrospective study of children diagnosed with CD in two paediatric centres demonstrates that the presence of granulomata at diagnosis was associated with disease phenotype, nutritional status and the subsequent disease severity (reflected by the need for immunosuppressive therapies). The presence of granulomata was not, however, associated with the development of disease complications (such as fistulising or stricturing disease), EIM or the presence of perianal disease in this cohort.

Overall, just over half of the children included in the current study had granulomata present at diagnosis. This rate is consistent with previous reports evaluating the presence of granulomata in children diagnosed with $C D$, with a reported frequency between $24.5 \%$ and $61 \%[10,12-14]$. Several factors appear to influence the presence of granulomata, while other factors may be relevant to the detection of granulomata. For instance, younger age at diagnosis has been associated with increased granuloma rate [10]. The number of mucosal biopsies obtained and the locale of biopsies (namely inclusion of upper endoscopy and biopsies rather than just ileo-colonoscopy) may increase the yield.

Granulomata were identified more often in children with isolated colonic involvement in the current study but not in those with associated perianal disease. Similarly, Rothschild et al. [15] noted that the presence of granulomata in their group of 289 Israeli children was not associated with perianal involvement. This report did, however, find that granulomata were more commonly associated with upper gut involvement and ileo-colonic disease. Other studies have shown similar associations between granulomata and disease location $[9,10,12]$, but have also demonstrated an association with perianal disease $[10,12,16-18]$. The reasons for these differences are unclear but may reflect the size of the populations included.

Previous studies have attempted to ascertain the relationship between the presence of granulomata and EIM. Two reports involving a similar number of subjects to the current study did not show any increase in EIM in association with granulomata [19]. In contrast, one study that included 289 patients with CD noted that the frequency of EIM in those without granulomata was twice that seen in those with granulomata [15]. This difference did not appear to be due to the length of follow-up from diagnosis, as each report (including the current study) had a disease duration of approximately eight years.

The children shown to have granulomata in the current report had lower weight $z$-scores at diagnosis than the children without granulomata. This relationship was not seen in two earlier paediatric studies [12,15]. Whilst age at diagnosis appeared similar between these studies, other factors 
that could affect nutritional status, such as duration of symptoms prior to diagnosis, disease extent at diagnosis and disease activity at diagnosis, were not directly available for comparison between the reports.

The current report showed an association between the presence of granulomata and some aspects of disease severity and outcomes (e.g., requirement for immunomodulatory therapies) but not other outcomes (e.g., EIMs, perianal disease). Other reports, including a meta-analysis that incorporated 19 studies, involving children or adults have generally shown that the presence of granulomata is linked to worse outcomes such as disease progression, hospital admissions and surgery [20-22]. Interestingly, one recent report also concluded that the presence of mucosal granulomata or mesenteric lymph node granulomata is associated with post-operative recurrence after resection (23).

The underlying reasons for the difference between the outcomes of individuals with CD according to the presence or absence of a granuloma are unknown. The diagnosis of CD includes various patterns of disease, that could each be seen as sub-types of CD. The presence of a granuloma may be part of this. Furthermore, the aetiology of granulomata in the setting of CD is not well understood. It is possible that differences in genetic risk factors or variations in levels of cytokines or other inflammatory mediators may influence the development of granuloma, and that these factors may be more critical in determining the disease course and outcome.

While this report included a small data set, this was felt to be consequent to the tight inclusion criteria. A larger data set may have enabled a clearer delineation of the relevance of granulomata in children diagnosed with CD. The data were comprised of two geographically distinct populations, with no differences in outcomes between the two groups. Further, the retrospective design of this report did not enable collection of potentially relevant data after diagnosis (such as growth or subsequent adult height acquisition, or subsequent histological assessments).

In conclusion, this study revealed interesting clues of a potential link between the presence of granulomata and certain key disease outcomes within a group of children diagnosed with CD. Although statistically significant results were obtained, the study included two small populations meaning that further associations may exist. Further studies involving larger patient cohorts with well-defined outcome variables are required to substantiate and build upon these preliminary findings. In conclusion, the histological presence of granulomata may provide important predictive information that might inform more personalised therapeutic strategies for individual patients.

Author Contributions: A.S.D., R.B.G., E.W. and L.A. conceived the study design. E.W. and F.J. collated and analysed initial data. L.A. contributed to data analysis, prepared figures and tables, and composed results. E.W. and L.A. jointly contributed to the preparation of the first draft of the manuscript. A.S.D. and R.H. provided supervision in the study sites. All authors contributed to the critical review of the manuscript and all have read and agreed to the published version of the manuscript.

Acknowledgments: We acknowledge the support of Cure Kids New Zealand. R.H. is supported by an NHS Research Scotland Career Researcher Fellowship. No other funding acknowledgements.

Conflicts of Interest: A.S.D. has received honoraria from Janssen and Abbvie. R.H. has received consultancy fees, speaker's fees, conference or travel support from 4D pharma and Nutricia. No other disclosures of direct conflict of interest.

\section{References}

1. Loddo, I.; Romano, C. Inflammatory bowel disease: Genetics, epigenetics, and pathogenesis. Front. Immunol. 2015, 6, 551. [CrossRef] [PubMed]

2. Zhang, Y.Z.; Li, Y.Y. Inflammatory bowel disease: Pathogenesis. World J. Gastroenterol. 2014, 20, 91-99. [CrossRef] [PubMed]

3. Rosen, M.J.; Dhawan, A.; Saeed, S.A. Inflammatory Bowel Disease in Children and Adolescents. JAMA Pediatr. 2015, 169, 1053-1060. [CrossRef] [PubMed]

4. Sykora, J.; Pomahacova, R.; Kreslova, M.; Cvalinova, D.; Stych, P.; Schwarz, J. Current global trends in the incidence of pediatric-onset inflammatory bowel disease. World J. Gastroenterol. 2018, 24, 2741-2763. [CrossRef] 
5. Pigneur, B.; Seksik, P.; Viola, S.; Viala, J.; Beaugerie, L.; Girardet, J.P.; Ruemmele, F.M.; Cosnes, J. Natural history of Crohn's disease: Comparison between childhood-and adult-onset disease. Inflamm. Bowel Dis. 2010, 16, 953-961. [CrossRef]

6. Van Limbergen, J.; Russell, R.K.; Drummond, H.E.; Aldhous, M.C.; Round, N.K.; Nimmo, E.R.; Smith, L.; Gillett, P.M.; McGrogan, P.; Weaver, L.T.; et al. Definition of phenotypic characteristics of childhood-onset inflammatory bowel disease. Gastroenterology 2008, 135, 1114-1122. [CrossRef]

7. Levine, A.; Koletzko, S.; Turner, D.; Escher, J.C.; Cucchiara, S.; de Ridder, L.; Kolho, K.L.; Veres, G.; Russell, R.K.; Paerregaard, A.; et al. ESPGHAN revised porto criteria for the diagnosis of inflammatory bowel disease in children and adolescents. J. Pediatr. Gastroenterol. Nutr. 2014, 58, 795-806. [CrossRef]

8. Ruemmele, F.M.; Veres, G.; Kolho, K.L.; Griffiths, A.; Levine, A.; Escher, J.C.; Amil Dias, J.; Barabino, A.; Braegger, C.P.; Bronsky, J.; et al. Consensus guidelines of ECCO/ESPGHAN on the medical management of pediatric Crohn's disease. J. Crohns Colitis 2014, 8, 1179-1207. [CrossRef]

9. Freeman, H.J. Natural history and long-term clinical course of Crohn's disease. World J. Gastroenterol. 2014, 20,31-36. [CrossRef]

10. Heresbach, D.; Alexandre, J.L.; Branger, B.; Bretagne, J.F.; Cruchant, E.; Dabadie, A.; Dartois-Hoguin, M.; Girardot, P.M.; Jouanolle, H.; Kerneis, J.; et al. Frequency and significance of granulomas in a cohort of incident cases of Crohn's disease. Gut 2005, 54, 215-222. [CrossRef]

11. Molnar, T.; Tiszlavicz, L.; Gyulai, C.; Nagy, F.; Lonovics, J. Clinical significance of granuloma in Crohn's disease. World J. Gastroenterol. 2005, 11, 3118-3121. [CrossRef] [PubMed]

12. De Matos, V.; Russo, P.A.; Cohen, A.B.; Mamula, P.; Baldassano, R.N.; Piccoli, D.A. Frequency and clinical correlations of granulomas in children with Crohn disease. J. Pediatr. Gastroenterol. Nutr. 2008, 46, 392-398. [CrossRef] [PubMed]

13. Jevon, G.P.; Madhur, R. Endoscopic and histologic findings in pediatric inflammatory bowel disease. Gastroenterol. Hepatol. (N Y) 2010, 6, 174-180.

14. Mazor, Y.; Karban, A.; Nesher, S.; Weiss, B.; Leshinsky-Silver, E.; Levine, A.; Eliakim, R. Granulomas in Crohn's disease: Are newly discovered genetic variants involved? J. Crohns Colitis 2010, 4, 438-443. [CrossRef]

15. Rothschild, B.; Rinawi, F.; Herman, Y.; Nir, O.; Shamir, R.; Assa, A. Prognostic significance of granulomas in children with Crohn's disease. Scand. J. Gastroenterol. 2017, 52, 716-721. [CrossRef] [PubMed]

16. Denoya, P.; Canedo, J.; Berho, M.; Allende, D.S.; Bennett, A.E.; Rosen, L.; Hull, T.; Wexner, S.D. Granulomas in Crohn's disease: Does progression through the bowel layers affect presentation or predict recurrence? Colorectal Dis. 2011, 13, 1142-1147. [CrossRef]

17. Markowitz, J.; Kahn, E.; Daum, F. Prognostic significance of epithelioid granulomas found in rectosigmoid biopsies at the initial presentation of pediatric Crohn's disease. J. Pediatr. Gastroenterol. Nutr. 1989, 9, 182-186. [CrossRef]

18. So, H.; Ye, B.D.; Park, Y.S.; Kim, J.; Kim, J.S.; Moon, W.; Lee, K.M.; Kim, Y.S.; Keum, B.; Kim, S.E.; et al. Gastric lesions in patients with Crohn's disease in Korea: A multicenter study. Intest. Res. 2016, 14, 60-68. [CrossRef]

19. Ramzan, N.N.; Leighton, J.A.; Heigh, R.I.; Shapiro, M.S. Clinical significance of granuloma in Crohn's disease. Inflamm. Bowel Dis. 2002, 8, 168-173. [CrossRef]

20. Hong, S.W.; Yoon, H.; Shin, C.M.; Park, Y.S.; Kim, N.; Lee, D.H.; Kim, J.S. Clinical Significance of Granulomas in Crohn's Disease: A Systematic Review and Meta-analysis. J. Gastroenterol. Hepatol. 2019, 35, 364-373. [CrossRef]

21. Johnson, C.M.; Hartman, D.J.; Ramos-Rivers, C.; Rao, B.B.; Bhattacharya, A.; Regueiro, M.; Schwartz, M.; Swoger, J.; Al Hashash, J.; Barrie, A.; et al. Epithelioid Granulomas Associate With Increased Severity and Progression of Crohn's Disease, Based on 6-Year Follow-Up. Clin. Gastroenterol. Hepatol. 2018, 16, 900-907. [CrossRef] [PubMed]

22. Watermeyer, G.; Thomson, S.R. Granulomas at initial diagnosis of Crohn's disease signal a poor outcome. S. Afr. Med. J. 2015, 105, 480-483. [CrossRef] [PubMed]

(C) 2020 by the authors. Licensee MDPI, Basel, Switzerland. This article is an open access article distributed under the terms and conditions of the Creative Commons Attribution (CC BY) license (http://creativecommons.org/licenses/by/4.0/). 